\title{
Self-consistent simulation of intermediate band solar cells: Effect of occupation rates on device characteristics
}

\author{
Katsuhisa Yoshida, ${ }^{1,2, a)}$ Yoshitaka Okada, ${ }^{1,2}$ and Nobuyuki Sano ${ }^{3}$ \\ ${ }^{1}$ Graduate School of Engineering, The University of Tokyo, 7-3-1 Hongo, Bunkyo-ku, Tokyo 113-8656, \\ Japan \\ ${ }^{2}$ Research Center for Advanced Science and Technology, The University of Tokyo, 4-6-1 Komaba, \\ Meguro-ku, Tokyo 153-8904, Japan \\ ${ }^{3}$ Institute of Applied Physics, University of Tsukuba, 1-1-1 Tennodai, Tsukuba, Ibaraki 305-8573, Japan
}

(Received 2 July 2010; accepted 21 August 2010; published online 28 September 2010)

\begin{abstract}
In order to design optimum structures for intermediate band solar cells, simulations based on self-consistent drift-diffusion model with a suitable treatment of the intermediate band in device domain are necessary. In this work, we have included the dependence of occupation rate of intermediate band at each position on optical generation rate via the intermediate band. Typical material parameters of GaAs were used except for the absorption coefficient of each corresponding band-to-band transition. Simulation results using our model indicate that the dependence of occupation rate on device position strongly affect short-circuit currents and also electrostatic potentials of the cell. (C) 2010 American Institute of Physics. [doi:10.1063/1.3488815]
\end{abstract}

In an intermediate band solar cell (IBSC), ${ }^{1}$ an additional current generation path by two-step absorption of subband gap photons via IB is introduced to a conventional singlejunction solar cell. The inclusion of an IB can lift the Shockley-Queisser efficiency limit of a single gap solar cell. $^{2}$

In order to realize IBSCs, multistacked quantum dots (QDs) or a three-dimensional QD superlattice, ${ }^{3-6}$ an impurity-band, ${ }^{7}$ and a band anticrossing in a mismatched alloy semiconductor ${ }^{8}$ have been proposed. The detailed balance model $^{1,9-11}$ and drift-diffusion method with an analytical expansion of the Poisson equation or current continuity equations ${ }^{12-15}$ have been used to theoretically investigate the optimal combination of IB states and energy band gap of host material, operation under concentration, and effect of overlapping spectra of absorption coefficients. However, the detailed balance approach does not incorporate any physical information of device structure and the latter is not a selfconsistent treatment. Recently, Lin, and Phillips have reported on a self-consistent drift-diffusion method incorporating the effect of carrier transport and recombination to analyze operation of IBSCs. ${ }^{16}$ However, the effect of occupation rate of IB states was treated as constant, though in practice, is dependent on the position.

In this letter, we adequately incorporate the effect of occupation rate of IB at each point on the optical generation rate via IB in IBSC using self-consistent drift-diffusion method. As GaAs based structure is widely studied for IBSCs ${ }^{3-6}$ we adopt material parameters ${ }^{17}$ of GaAs at $300 \mathrm{~K}$ except for the absorption coefficients, and the same treatment of radiative recombination rates reported in Ref. 16 is used. We neglect electron transport through IB states and an infinite surface recombination velocity is assumed as the boundary condition. The effective density of IB states is set to be $N_{I}=5.0 \times 10^{16} \mathrm{~cm}^{-3}$, and a half of $N_{I}$ is the actual density of QDs. The absorption coefficients $\alpha_{C V}, \alpha_{C I}, \alpha_{I V}$ are assumed non-overlapping and $1.0 \times 10^{4} \mathrm{~cm}^{-1}$ in each spectrum. The

${ }^{\text {a)} E l e c t r o n i c ~ m a i l: ~ y o s h i d a @ m b e . r c a s t . u-t o k y o . a c . j p . ~}$ energy position of IB states is $\mathrm{E}_{I V}=0.95 \mathrm{eV}$ above the valence band (VB) edge such that this energy position divides the number of subband gap photons in solar spectrum into roughly two, from $\mathrm{E}_{C I}=0.48 \mathrm{eV}$ to $\mathrm{E}_{C V}=1.43 \mathrm{eV}$.

The optical generation rate from VB to conduction band (CB) along the position $x$ is defined by

$$
G_{C V}(x)=\int_{E_{C V}}^{\infty} \alpha_{C V} F_{0}(E) \exp \left(-\alpha_{C V} x\right) d E,
$$

where $F_{0}(E)$ is the incident light from nonconcentrated solar spectrum of energy $E$ given by a black body radiation spectrum at $5800 \mathrm{~K} . \alpha_{C V}$ is the absorption coefficient for CB-VB transitions. For optical generation via IB, the IB state works as an initial state of IB to $\mathrm{CB}$ transition and also as a final state for VB to IB optical transition. In the case of dispersion-less relation with the wave number and $E_{I}$, net absorption coefficient for $\mathrm{CB}-\mathrm{IB}$ and $\mathrm{IB}-\mathrm{VB}$ transitions which depend on carrier occupation in IB states can be expressed as $\alpha_{C I} \times f_{I}(x)$ and $\alpha_{I V} \times\left\{1-f_{I}(x)\right\}$, where $\alpha_{C I}$ and $\alpha_{I V}$ are the absorption coefficients for CB-IB and IB-VB optical transitions and $f_{I}(x)$ is the occupation rate at position $x$, respectively. The net absorption coefficient is defined as an absorption rate of incident spectrum per unit length. Thus the optical generation rate for CB-IB transition can be defined as

$$
\begin{aligned}
& G_{C I}(x)=\int_{E_{C I}}^{E_{I V}} \alpha_{C I} f_{I}(x) F_{0}(E) \exp \left[-\alpha_{C I} x_{C I}(x)\right] d E, \\
& x_{C I}(x)=\int_{x_{0}}^{x} f_{I}\left(x^{\prime}\right) d x^{\prime},
\end{aligned}
$$

where $x_{C I}(x)$ is the effective absorption length at a position $x$ for CB-IB optical generation rate. The subband gap photons are absorbed by IB states, and $x_{0}$ correspond to the left edge of IB region. Similarly, the optical generation rate of IB-VB transition is expressed by 

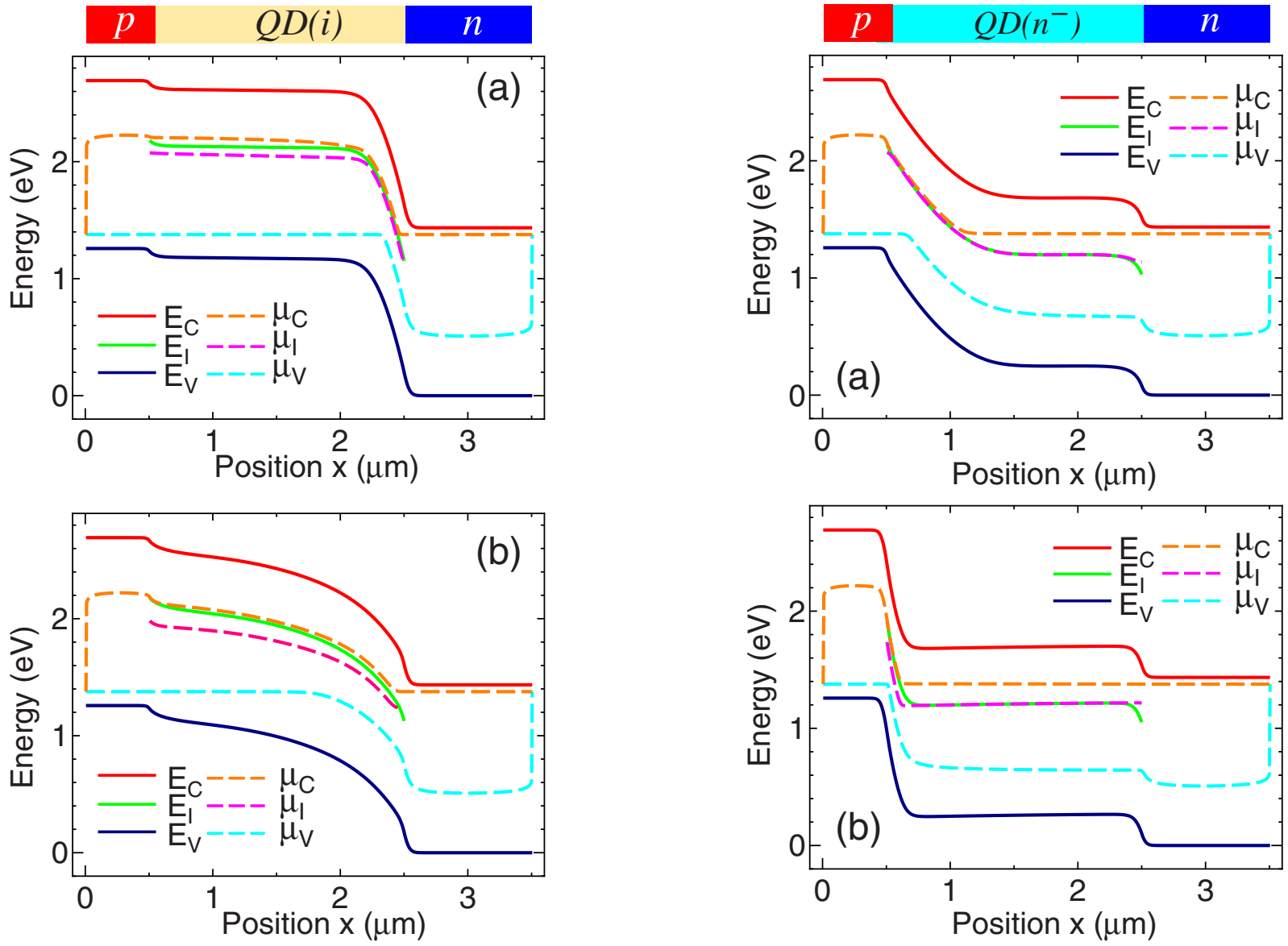

FIG. 1. (Color online) Calculated band diagrams for the case that the IB region is intrinsic. (a) $G_{C I}$ and $G_{I V}$ both depend on $f_{I}$ and (b) independent of $f_{I}$, respectively. $E_{C}, E_{I}$, and $E_{V}$ are $\mathrm{CB}, \mathrm{IB}$, and $\mathrm{VB}$ energies, and $\mu_{C}, \mu_{I}$, and $\mu_{V}$ are quasi-Fermi energies of $\mathrm{CB}, \mathrm{IB}$, and $\mathrm{VB}$, respectively.

$$
G_{I V}(x)=\int_{E_{I V}}^{E_{C V}} \alpha_{I V}\left\{1-f_{I}(x)\right\} F_{0}(E) \exp \left[-\alpha_{I V} x_{I V}(x)\right] d E,
$$

where $x_{I V}(x)$ is the effective absorption length at a position $x$ for IB-VB optical generation rate and is described by

$$
x_{I V}(x)=\int_{x_{0}}^{x}\left\{1-f_{I}\left(x^{\prime}\right)\right\} d x^{\prime} .
$$

The CB-IB and IB-VB optical generation rates are substituted into the balance equation for electrons in IB with the radiative recombination rate of $\mathrm{CB}-\mathrm{IB}, U_{C I}(x)$, and of $\mathrm{IB}-$ $\mathrm{VB}, U_{I V}(x)$, at each position in IB region,

$$
G_{C I}(x)-U_{C I}(x)=G_{I V}(x)-U_{I V}(x) .
$$

The left terms are included in the continuity equation for electrons in $\mathrm{CB}$ and the right terms are included in the continuity equation for holes. The carrier concentration is determined self-consistently by using the Poisson equation, the continuity equations, and the balance equation of IB.

We first calculated the band diagrams in the short-circuit condition. The accepter density in $p$-emitter (top layer) is $7.0 \times 10^{16} \mathrm{~cm}^{-3}$ and the donor density in $n$-base (bottom layer) is taken to be $5.0 \times 10^{16} \mathrm{~cm}^{-3}$. The calculated energy band diagrams for the case of IB region being intrinsic (undoped) is shown in Fig. 1, and for doped n-type with the same density as QDs is shown in Fig. 2, respectively. In Figs.

FIG. 2. (Color online) Calculated band diagrams for the case that the IB region is doped with donor density equal to the density of QDs. (a) Generation rates $G_{C I}$ and $G_{I V}$ both depend on $f_{I}$, and (b) independent of $f_{I}$, respectively.

1(b) and 2(b) are for the case of constant optical generation rate $f_{I}(x)$ via IB. In this case, the effective absorption lengths $x_{C I}(x)$ and $x_{I V}(x)$ are simply expressed as $x-x_{0}$ in IB region. The quasi-Fermi energy of IB, $\mu_{I}(x)$, is estimated by fitting the occupation rate of IB by Fermi-Dirac function at each position. In this sense, the profile of $\mu_{I}$ expresses the information on IB occupation rate. The energy band diagrams are significantly different if the optical generation rate via IB is taken to vary with $x$ as shown in these figures.

The optical generation rate via IB as a function of position of IB region are plotted in Fig. 3. For the case of independent $f_{I}$ (solid curves), the rates show a simple exponentially decrease along the position. On the contrary, for the case of $G_{C I}$ and $G_{I V}$ which depend on $f_{I}(x)$ (dashed curves) show a more complex behavior. Near the $p$-emitter side of IB region, $G_{I V}(x)$ has a larger value than $G_{C I}(x)$. This is because the IB occupation rates are smaller than 0.5 . This implies that there is a larger chance that the electrons in VB are excited to IB states. On the other hand, near the $n$-emitter side of IB region, the situation is reversed and $G_{C I}(x)$ is now greater than $G_{I V}(x)$. In the middle of IB region, the occupation rates are almost 0.5 , and thus $G_{C I}(x)$ and $G_{I V}(x)$ have similar values. The difference of $G_{C I}(x)$ and $G_{I V}(x)$ at each position is approximately regarded as the amount of recombination rate by requirement from Eq. (6). Therefore, the matching of two optical generation rates, $G_{C I}(x)$ and $G_{I V}(x)$, is the key to maximize the additional current contributed by IB. The IB 


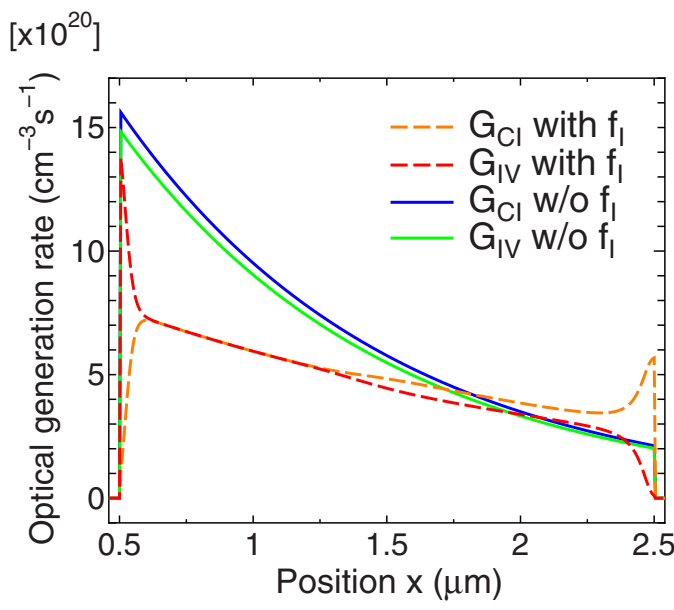

FIG. 3. (Color online) Optical generation rates via IB in intrinsic IB region case for the short-circuit condition. Dashed curves are $f_{I}$ dependent case and solid curves are $f_{I}$ independent case, respectively.

states and optimal occupied condition of IB states for IBSC can therefore be determined.

The current-voltage characteristics using our model are shown in Fig. 4. The value of short circuit current of $f_{I}$ dependent $p-i-n$ structure agrees with experimental results. ${ }^{6}$ The introduction of IB states into the conventional singlejunction solar cell (dotted-dashed curve), acts to increase the short-circuit currents as a consequence of two photons absorption via IB. In the present study, because we only account for radiative recombinations, the open-circuit voltage is not affected by the presence of IB states. The same consideration is reported in Ref. 16 and this corresponds to carrier lifetimes on the order of $1 \mu \mathrm{s}$ in a practical device. If IB region is doped, both $f_{I}$ dependent and independent cases result in large current enhancements by IB states. In these cases, IB occupation rates are almost 0.5 over the whole IB region. On the other hand, if IB region is intrinsic, for both $f_{I}$ dependent model and independent model, the IB occupation rate is lower than 0.5 , and does not satisfy the half-filled

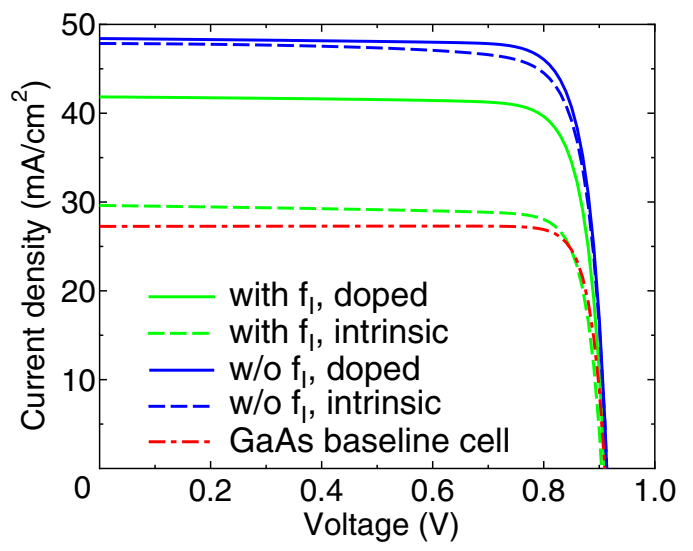

FIG. 4. (Color online) Current-voltage characteristics. Darker dashed and solid lines are independent $f_{I}$ of $G_{C I}$ and $G_{I V}$. Lighter lines are dependent. Dotted-dashed line is GaAs baseline cell without IB states. condition. ${ }^{15}$ However, $f_{I}$ independent model gives almost the same short-circuit current value as for doped IB region case. The difference in I-V characteristics is affected by the electrostatic potential profile, mobility model of carriers, and electric field. However, in $f_{I}$ dependent model, the enhancement of short-circuit current by introduction of IB is very small. This is because the occupation rates of IB states in this case are very small. The requirement of Eq. (6) suggests that a large difference of $G_{I V}$ and $G_{C I}$ leads to a large recombination rate of $U_{I V}$. The maximum enhanced short-circuit current is obtained by $J_{b s}+J_{I B}$. $J_{b s}$ is short-circuit current without IB states and depends on the boundary conditions, $J_{I B}$ is limited by whichever $J_{C I} \times\left[1-\exp \left(-\alpha_{C I} x_{C I}\right)\right]$ or $J_{I V} \times[1$ $\left.-\exp \left(-\alpha_{I V} x_{I V}\right)\right]$ has a smaller value. The average value of IB occupation rate is 0.07 in Fig. 1(a) and 0.5 in Fig. 2(a). This gives $J_{b s}=27.3 \mathrm{~mA} / \mathrm{cm}^{2}$ and $J_{I B}=3.6 \mathrm{~mA} / \mathrm{cm}^{2}$ for Fig. 1(a) and $J_{I B}=15.1 \mathrm{~mA} / \mathrm{cm}^{2}$ in Fig. 2(a), respectively. These estimated values agree well with the calculated values shown in Fig. 4.

In conclusion, we have developed a self-consistent driftdiffusion method with IB balance equations for IB electrons, and considered $f_{I}$ dependence of $G_{C I}$ and $G_{I V}$ at each IB position. The introduction of $f_{I}$ dependent optical generation rates via IB clarifies the relation of occupation rates of IB states and short circuit current and affects to electrostatic potential profiles. To design optimal structures for IBSCs, consideration of a more realistic treatment of absorption coefficients and nonradiative recombination rates will be included in our future calculation. Further, a self-consistent treatment of electron transport in IB should also be included.

The authors acknowledge funding from New Energy and Technology Development Organization (NEDO), and Ministry of Economy, Trade, and Industry (METI), Japan.

${ }^{1}$ A. Luque and A. Martí, Phys. Rev. Lett. 78, 5014 (1997).

${ }^{2}$ W. Shockley and H. J. Queisser, J. Appl. Phys. 32, 510 (1961).

${ }^{3}$ S. M. Hubbard, C. D. Cress, C. G. Bailey, R. P. Raffaelle, S. G. Bailey, and D. M. Wilt, Appl. Phys. Lett. 92, 123512 (2008).

${ }^{4}$ V. Popescu, G. Bester, M. Hanna, A. G. Norman, and A. Zunger, Phys. Rev. B 78, 205321 (2008).

${ }^{5}$ R. Oshima, A. Takata, and Y. Okada, Appl. Phys. Lett. 93, 083111 (2008).

${ }^{6}$ Y. Okada, R. Oshima, and A. Takata, J. Appl. Phys. 106, 024306 (2009).

${ }^{7}$ E. Antolín, A. Martí, J. Olea, D. Pastor, G. González-Díaz, I. Mártil, and A. Luque, Appl. Phys. Lett. 94, 042115 (2009).

${ }^{8}$ W. Wang, A. S. Lin, and D. Phillips, Appl. Phys. Lett. 95, 011103 (2009).

${ }^{9}$ L. Cuadra, A. Martí, and A. Luque, IEEE Trans. Electron Devices 51, 1002 (2004).

${ }^{10}$ M. Y. Levy and C. Honsberg, Phys. Rev. B 78, 165122 (2008).

${ }^{11}$ R. Strandberg and T. W. Reenaas, J. Appl. Phys. 105, 124512 (2009).

${ }^{12}$ A. Martí, L. Cuadra, and A. Luque, IEEE Trans. Electron Devices 49, 1632 (2002).

${ }^{13}$ A. Luque, A. Martí, N. López, E. Cánovas, C. Sranley, C. Farmer, and P. Díaz, J. Appl. Phys. 99, 094503 (2006).

${ }^{14}$ T. S. Navruz and M. Saritas, Sol. Energy Mater. Sol. Cells 92, 273 (2008).

${ }^{15}$ A. S. Lin, W. Wang, and D. Phillips, J. Appl. Phys. 105, 064512 (2009).

${ }^{16}$ A. S. Lin and J. D. Phillips, IEEE Trans. Electron Devices 56, 3168 (2009).

${ }^{17} \mathrm{~S}$. Selberherr, Analysis and Simulation of Semiconductor Devices (Springer, Vienna, 1984). 